\title{
MECHANISTIC MODELING OF STRENGTH DISTRIBUTION OF QUASIBRITTLE STRUCTURES AND ITS IMPLICATION FOR STRUCTURAL RELIABILITY
}

\author{
JIA-LIANG LE* AND ZDENĚK P. BAŽANT ${ }^{\ddagger}$ \\ *University of Minnesota \\ Minneapolis, MN, USA \\ e-mail: jle@umn.edu \\ ${ }^{\ddagger}$ Northwestern University \\ Evanston, IL, USA \\ e-mail: z-bazant@northwestern.edu
}

Key words: Weakest link model, Strength statistics, Size effect, Reliability indices, Safety factors

\begin{abstract}
This paper reviews a recently developed finite weakest link model of strength of quasibrittle structures, which fail under controlled load at macro-crack initiation from one representative volume element (RVE). The probability distribution of RVE strength is derived from the transition state theory and a hierarchical multi-scale transition model. The model predicts that the strength distribution of quasibrittle structures depends on the structure size and geometry, transitioning from a predominantly Gaussian distribution to a Weibull distribution as the structure size increases. By further considering the load randomness, it is shown that the size dependence of the strength distribution directly leads to a strong size effect on the Cornell reliability index. Through asymptotic matching, approximate size effect equations can be constructed for both the nominal and central safety factors. The important role of size effect in the reliability analysis of large-size structures is demonstrated through the analysis of the failure of the Malpasset Dam.
\end{abstract}

\section{INTRODUCTION}

It is a well accepted fact that no engineering structures can be designed to be risk free. In practice, the design of structures is required to target a tolerable failure probability, which is generally on the order of $10^{-6}[17,27,28]$. For structural design against such a low failure risk, a good understanding of the probability distribution of structural strength is essential. For perfectly ductile and brittle structures, the strength distribution can be determined from well-established probability theories. The cumulative distribution function (cdf) of strength of ductile structures must follow the Gaussian distribution according to the Central Limit The- orem [1] since the nominal structural strength can be calculated as the weighted sum of the strengths of the material elements along the failure surface. By contrast, the failure of perfectly brittle structures is triggered by the failure of one material element whose size is negligible compared to the structure size. According to the classical weakest link model, the strength cdf of brittle structures must follow the Weibull distribution [32,33]. Both the Gaussian and Weibull distributions are two-parameter probability distribution functions, which can be calibrated by histogram testing of a relatively small number of specimens.

Neither of the aforementioned cases can be 
applied to quasibrittle structures, in which the size of material inhomogenieties is not negligible compared to the overall structure size. As a result, the failure behavior of quasibrittle structures lies in between the ductile and brittle limits. Furthermore, it has been well documented that failure of quasibrittle structures exhibits a strong size dependence, transitioning from a quasi-plastic failure behavior for smallsize structures to a perfectly brittle behavior for large-size structures [3-5, 10]. Based on the aforementioned probability distributions of strengths of ductile and brittle structures, it is clear that the strength cdf is closely tied with the failure behavior of the structure. Since quasibrittle structures have a size-dependent failure mechanism, it is natural to expect that the strength cdf would depend on the structure size and geometry.

This paper reviews a recently developed probabilistic model of strength of quasibrittle structures with an emphasis on its size dependence [6, 8, 9, 23]. This probabilistic model has important implications on reliability-based design of quasibrittle structures in terms of the size dependence of reliability indices and safety factors, which is also summarized in this paper.

\section{FINITE WEAKEST LINK MODEL}

This study focuses on the quasibrittle structures, which fail (under controlled load) at the initiation of a macro-crack from one RVE. This is a dangerous failure mode since there is no precursor to the failure. Statistically, the failure of this class of structures is equivalent to a chain of RVEs and follows the weakest-link model, in which each link corresponds to one RVE and has a statistically independent strength [18]. Consequently, the strength distribution of the structure can be calculated by using the joint probability theorem, i.e.:

$$
P_{R}\left(\sigma_{N}\right)=1-\Pi_{i=1}^{N}\left\{1-P_{1}\left[\sigma_{N} s\left(\boldsymbol{x}_{i}\right)\right]\right\}
$$

where $N=$ number of RVEs in the structure $=V / V_{0}, V$ and $V_{0}$ are the volumes of the entire structure and one RVE, respectively, $P_{1}=$ cdf of strength of one RVE, $\sigma_{N}=$ nominal structural strength, which is a load parameter of the dimension of stress. In general, $\sigma_{N}=c_{n} P_{m} / b D$ or $c_{n} P_{m} / D^{2}$ for two- or threedimensional scaling, $P_{m}=$ maximum load of the structure, $c_{n}=$ dimensionless parameter chosen such that $\sigma_{N}$ represents the maximum principal stress in the structure, $b=$ structure thickness in the third dimension, $D=$ characteristic structure dimension or size, and $s\left(\boldsymbol{x}_{i}\right)=$ dimensionless stress field such that the actual stress $\sigma\left(\boldsymbol{x}_{i}\right)$ in the $i$-th RVE centered at coordinates $\boldsymbol{x}_{i}$ is equal to $\sigma_{N} s\left(\boldsymbol{x}_{i}\right) . \sigma\left(\boldsymbol{x}_{i}\right)$ may be interpreted as the maximum principal stress $\sigma_{I}$.

More generally, stress $\sigma$ may be replaced by a suitable stress tensor function, $c(\boldsymbol{\sigma})$, capturing the triaxiality of failure criterion [10, 29]. This function can be simplified into the condition of independent survival of the RVE under each of the three principal stresses, $\sigma_{I}, \sigma_{I I}$ and $\sigma_{I I I}$ [12], provided that a crack normal to any principal stress can cause failure. In this study the second and third principal stresses are assumed to be either correlated to the first or too small compared to the first $\sigma\left(\boldsymbol{x}_{i}\right)=c\left(\boldsymbol{\sigma}\left(\boldsymbol{x}_{i}\right)\right) \approx$ $\sigma_{I}\left(\boldsymbol{x}_{i}\right)$, in which Eq. 1 can be re-written as

$$
\begin{aligned}
\ln \left(1-P_{R}\right) & =\sum_{i=1}^{N} \ln \left[1-P_{1}\left(c\left(\boldsymbol{\sigma}\left(\boldsymbol{x}_{i}\right)\right)\right)\right](2) \\
& \left.\approx \sum_{i=1}^{N} \sum_{j=I}^{I I I} \ln \left[1-P_{1}\left(\sigma_{j}\left(\boldsymbol{x}_{i}\right)\right)\right\} \beta\right) \\
& \approx \sum_{i=1}^{N} \ln \left[1-P_{1}\left(\sigma_{I}\left(\boldsymbol{x}_{i}\right)\right)\right]
\end{aligned}
$$

It is clear that for structure of any size and geometry the calculation of the overall failure probability hinges on the knowledge of the strength distribution of one RVE. In a series of recent studies [6, 8, 9, 23], a multiscale statistical model was developed to derive the functional form of the cdf of RVE strength (Fig. 1). In this model, the failure probability of a nanoscale structural element, such as an atomic lattice and a disordered system of nano-particles, is first determined based on the Kramer's rule of transition state theory and on atomistic fracture mechanics (Fig. 1a) [6, 21, 23]. 
To link the failure statistics of the nano-scale structural element to the macroscopic RVE, a hierarchical statistical model is formulated, which consists of a bundle of only two long subchains, each of which consists of sub-bundles of two sub-sub-chains, each of which consists of sub-sub-bundles, etc., until the nano-scale element is reached (see Fig. 1b). Though the hierarchical model merely represents a mathematical approximation of multi-scale transition of strength statistics, it qualitatively reflects two main physical mechanisms of the failure of quasibrittle materials, namely the distributed damage (as a condition of compatibility of deformations of the sub-RVEs along the FPZ) and the localization of damage (among the sub-RVEs across the FPZ).

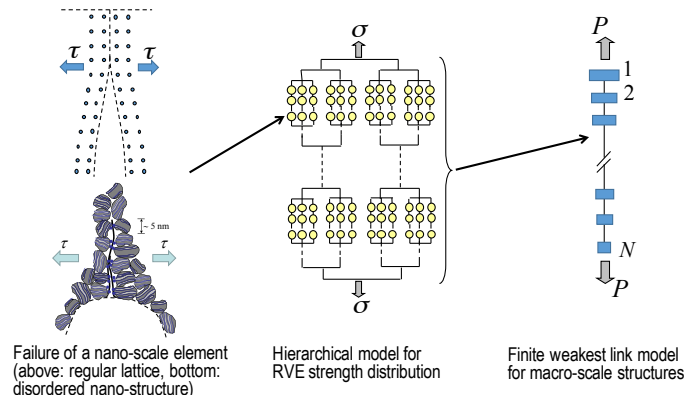

Figure 1: Multiscale modeling of strength statistics of quasibrittle structures.

Based on the properties of the strength statistics of bundles and chains, it has been shown that the cdf of RVE strength could be approximated by a Gaussian distribution onto which a Weibull tail is grafted at a probability about $10^{-4}-10^{-3}$. Mathematically, $P_{1}(\sigma)$ can be written as

$$
\begin{aligned}
P_{1}(\sigma)=1 & -e^{-\left\langle\sigma / s_{1}\right\rangle^{m}} \quad\left(\sigma \leq \sigma_{g r}\right) \\
P_{1}(\sigma)=P_{g r} & +\frac{r_{f}}{\delta_{G} \sqrt{2 \pi}} \\
& \times \int_{\sigma_{g r}}^{\sigma} e^{-\frac{\left(\sigma^{\prime}-\mu_{G}\right)^{2}}{2 \delta_{G}^{2}}} \mathrm{~d} \sigma^{\prime} \quad\left(\sigma>\sigma_{g r}\right)
\end{aligned}
$$

where $\langle x\rangle=\max (x, 0), m=$ Weibull modulus, $s_{0}=$ scale parameter of the Weibull tail,
$\mu_{G}$ and $\delta_{G}$ are the mean and standard deviation of the Gaussian core if considered extended to $-\infty ; r_{f}$ is a scaling parameter required to normalize the grafted cdf such that $P_{1}(\infty)=1, P_{g r}$ $=$ grafting probability $=1-\exp \left[-\left(\sigma_{g r} / s_{0}\right)^{m}\right]$, and $\sigma_{g r}=$ grafting stress. Finally, continuity of the probability density function (pdf) at the grafting point requires that $\left.\left(\mathrm{d} P_{1} / \mathrm{d} \sigma\right)\right|_{\sigma_{g r}^{+}}=$ $\left.\left(\mathrm{d} P_{1} / \mathrm{d} \sigma\right)\right|_{\sigma_{g r}^{-}}$.

Substitution of Eqs. 5a and 5b into Eq. 1 directly yields the probability distribution of structural strength $P_{R}\left(\sigma_{N}\right)$. We may consider $P_{R}\left(\sigma_{N}\right)$ to consist of two parts: the lower segment can be represented by a chain of Weibull elements, which must follow a Weibull distribution, and the upper segment can be represented by a chain of Gaussian elements. These two segments are separated at a probability $P_{g}=$ $1-\left(1-P_{g r}\right)^{N_{e q}}$, and $N_{e q}$ is the equivalent number of RVEs in the structure, which can be calculated as

$$
N_{e q}=\int_{V}\langle s(\boldsymbol{x})\rangle^{m} \mathrm{~d} V(\boldsymbol{x}) / V_{0}
$$

where $V_{0}=l_{0}^{n}, l_{0}=$ RVE size, and $n(=$ $1,2,3)=$ dimensionality of the structure. $N_{e q}$ physically represents the number of RVEs under uniform stress $\sigma_{N}$, which has the same cdf of the structural strength as does Eq. 1 1 under the assumption that the entire strength cdf follows the Weibull distribution [8,9].

Depending on the value of $N_{e q}$, three asymptotic behaviors of $P_{R}\left(\sigma_{N}\right)$ can be identified [23]:

1) When $N_{e q}$ is small, i.e. $N_{e q} \in(1,20)$, $P_{R}\left(\sigma_{N}\right)$ is predominantly Gaussian.

2) For the intermediate range of $N_{e q}$ values, i.e. $N_{e q} \in(50,500)$, the majority part of $P_{R}\left(\sigma_{N}\right)$ can be modeled as a chain of Gaussian of elements, which asymptotically converges to the Gumbel distribution [20].

3) For structures with a very large value of $N_{e q}$, i.e. $N_{e q} \geq 2000$, what matters for $P_{R}\left(\sigma_{N}\right)$ is the tail part of $P_{1}(x)$ and therefore Eq. 1 naturally approaches the Weibull distribution [32]. It should be pointed out that the intermediate asymptote of the Gumbel distribution is usually 
irrelevant because the small and large asymptotes are not too far from each other.

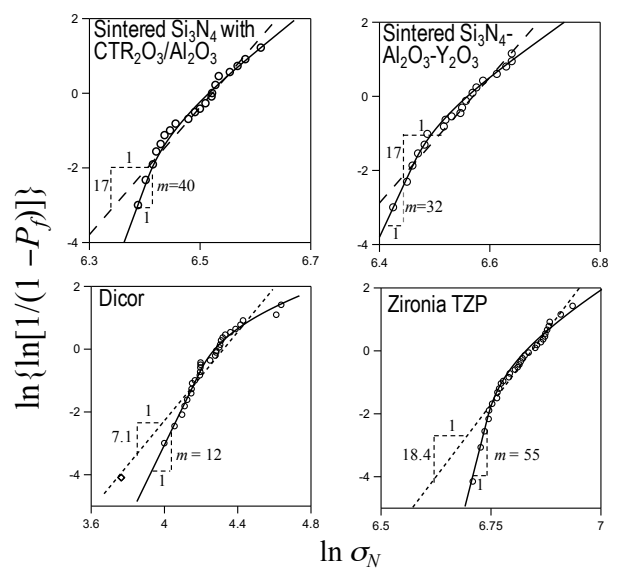

Figure 2: Optimum fitting of measured strength histograms by finite weakest link model.

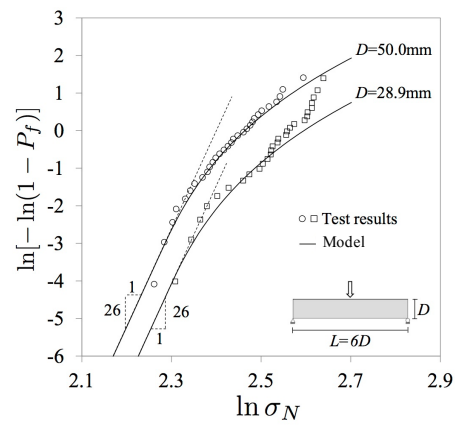

Figure 3: Size effect on strength distribution of asphalt mixtures [24].

The finite weakest link model has been validated by optimum fitting of measured strength histograms of many quasibrittle materials, such as engineering ceramics (sintered $\mathrm{Si}_{3} \mathrm{~N}_{4}$ with $\mathrm{CTR}_{2} \mathrm{O}_{3} / \mathrm{Al}_{2} \mathrm{O}_{3}$ [19] and sintered $\mathrm{Si}_{3} \mathrm{~N}_{4}$ with $\mathrm{Al}_{2} \mathrm{O}_{3}-\mathrm{Y}_{2} \mathrm{O}_{3}$ [30]) and dental ceramics (Dicor [31] and Zironia TZP [31]) (Fig. 2). In a recent study, a systematic size effect test was performed on the strength distribution of asphalt mixtures under three-point bending at a low temperature $\left(T=-24^{\circ}\right)$ [24]. Fig. 3 presents the measured strength distributions of beams of two different sizes. It is clear that the strength distribution exhibits a strong size effect, and such size dependence can be well captured by the present finite weakest link model.

\section{SIZE DEPENDENCE OF CORNELL RELIABILITY INDEX}

The foregoing discussion is limited to the strength statistics of structures. In general analysis of structural reliability, it is essential to also consider the uncertainty of applied loads. We may define the failure state of a quasibrittle structure as $Z=\sigma_{N}-\sigma_{L}<0$, where $\sigma_{L}=c_{n} P / b D=$ applied nominal stress of the structure. By noting that $\sigma_{N}$ and $\sigma_{L}$ are statistically independent, we can calculate the structural failure probability as

$$
P_{f}=\int_{0}^{\infty} P_{R}(x) f_{L}(x) \mathrm{d} x
$$

where $f_{L}(x)$ is the pdf of $\sigma_{L}$, and the cdf of structural strength $P_{R}(x)$ can be computed from Eq. 1. The most widely used approach to evaluate $P_{f}$ is through the Cornell reliability index within the framework of the First-Order Reliability Method (FORM) [1, 13, 14]. For a linear failure state, the Cornell index can be calculated as

$$
\beta=\left(\mu_{R}-\mu_{L}\right) / \sqrt{\delta_{R}^{2}+\delta_{L}^{2}}
$$

where $\mu_{R}, \mu_{L}=$ mean values of the structural strength $\sigma_{N}$ and the applied nominal stress $\sigma_{L}$, respectively, and $\delta_{R}, \delta_{L}=$ standard deviations of $\sigma_{N}$ and $\sigma_{L}$, respectively. If both $\sigma_{N}$ and $\sigma_{L}$ follow the Gaussian distributions, then the Cornell index can be directly used to compute the exact failure probability of the structure, i.e. $P_{f}=\Phi(-\beta)$, where $\Phi(x)=$ $(\sqrt{2 \pi})^{-1} \int_{\infty}^{x} e^{-x^{2} / 2} \mathrm{~d} x=$ standard Gaussian distribution function.

Based on Eq. 8, the dependence of the Cornell index can directly be related to the size effects on the mean and variance of $\sigma_{N}$, which can be computed for any set of geometrically similar structures as:

$\begin{aligned} \mu_{R}(D) & =\int_{0}^{\infty}\left[1-P_{R}\left(\sigma_{N}, D\right)\right] \mathrm{d} \sigma_{N} \\ \delta_{R}^{2}(D) & =\int_{0}^{\infty} \sigma_{N}^{2} \mathrm{~d} P_{R}\left(\sigma_{N}, D\right)-\mu_{R}^{2}(D)\end{aligned}$

Although closed-form evaluations of these integrals are not possible, approximate solutions for $\mu_{R}(D)$ and $\delta_{R}^{2}(D)$ can be constructed through 
asymptotic matching [7, 9, 22, 23]:

$$
\begin{aligned}
& \mu_{R}(D)=\left[\frac{N_{a}}{D}+\left(\frac{N_{b}}{D}\right)^{n r / m}\right]^{1 / r} \\
& \delta_{R}^{2}(D)=\left[\frac{C_{a}}{D}+\left(\frac{C_{b}}{D}\right)^{2 q n / m}\right]^{1 / q}
\end{aligned}
$$

where $N_{a}, N_{b}, r, C_{a}, C_{b}, q=$ model parameters. These parameters can be related to the statistical properties of RVE strength by the asymptotic conditions [22-24].

Knowing the model parameters for the approximate scaling relations for the mean and variance of $\sigma_{N}$, we readily obtain a sizedependent Cornell index for quasibrittle structures, i.e.:

$$
\beta=\frac{\left[\left(N_{a} / D\right)+\left(N_{b} / D\right)^{r n / m}\right]^{1 / r}-\mu_{L}}{\sqrt{\left[\left(C_{a} / D\right)+\left(C_{b} / D\right)^{2 q n / m}\right]^{1 / q}+\delta_{L}^{2}}}
$$

The size dependence of the Cornell index directly indicates that the overall failure probability of quasibrittle structures must depend on the structure size.

Note that, for quasibrittle structures, the Cornell index would not yield the exact failure probability (i.e. $\Phi(-\beta) \neq P_{f}$ ) since at least one probability distribution, namely that of $\sigma_{N}$, is generally not Gaussian. Nevertheless, the Cornell index provides a qualitative description of the risk level [16] and, as will be shown later, $\Phi(-\beta)$ can in some cases give a reasonable approximation of the failure probability for quasibrittle structures.

It should be pointed out that a recent study also investigated the size effect on the HasoferLind index, which provides a more robust way to compute the failure probability of quasibrittle structures. The details of the size effect analysis of the Hasofer-Lind index can be found in [22].

\section{CALCULATION OF SAFETY FAC- TORS}

The reliability index provides an efficient way of evaluating the failure risk of structures.
This lays down the foundation for the development of reliability-based design method for engineering structures. The most common approach for reliability-based structural design is to use the safety factors, in which the failure state is expressed by using either the nominal values or the mean values of the structural strength and nominal applied stress.

For example, if the mean values are used, the failure domain can be written as $\mu_{R}<\bar{\zeta} \mu_{L}$, where $\bar{\zeta}$ is referred to as the central safety factor, and if the nominal values are used, the failure domain becomes $\mu_{R N}<\zeta \mu_{L N}$, where $\mu_{R N}=$ nominal value of $\sigma_{N}=\mu_{R}-k_{R} \delta_{R}, \mu_{L N}=$ nominal value $=\sigma_{L}=\mu_{L}+k_{L} \delta_{L}$; here $k_{R}, k_{L}=$ constants, and $\zeta$ is the nominal safety factor.

Both the central and nominal safety factors $\zeta, \bar{\zeta}$ have to be determined to ensure that the overall failure probability of the structure is equal to a prescribed value $P_{c}$, which is typically on the order of $10^{-6}$. As discussed in the previous section, the overall failure probability of a quasibrittle structure strongly depends on the structure size. Therefore, we expect that the safety factors that correspond to a prescribed failure probability would also be size dependent. In this section, we investigate this size dependence by considering that the applied nominal stress follows a Gaussian distribution, which is a often assumed for typical loading, although a lognormal distribution might be more appropriate for bridges [15].

Based on the finite weakest link model, the probability distribution of $\sigma_{N}$ can be determined by four parameters of the strength cdf of one RVE (Eqs. 5a and b), i.e., the Weibull modulus $m$, Weibull scaling parameter $s_{0}$, mean RVE strength $\mu$, and coefficient of variation $(\mathrm{CoV})$ of RVE strength $\omega$. Here we fix the values of $m, \omega$ as well as the ratio between $\mu$ and $s_{0}$, denoted by $\psi$, and we will determine $s_{0}$ for a prescribed structural failure probability $P_{c}$.

To compute the safety factors, it is convenient to consider an artificial RVE, where the grafted strength cdf has the following parameters: Weibull modulus $=m$, Weibull scaling parameter $s_{0}=1$, mean strength $=\psi$, and 
$\mathrm{CoV}=\omega$. Since the Weibull tail of the grafted cdf of RVE strength is very short, the mean of the Gaussian core $\mu_{G}$ for this artificial material RVE is approximately equal to the mean RVE strength $\psi$ and the standard deviation of the Gaussian core $\delta_{G}$ is approximately equal to $\psi \omega$.

For a given structure, let $\mu_{r}, \delta_{r}$ denote the mean and standard deviation of the strength cdf by assuming that the structure is made of the aforementioned artificial RVE. Therefore, the mean and standard deviation of the strength cdf of the actual structure are simply equal to $s_{0} \mu_{r}$ and $s_{0} \delta_{r}$, respectively. The central and nominal safety factors of the structure can be computed as

$$
\begin{aligned}
\bar{\zeta}(D) & =\frac{s_{0}(D) \mu_{r}(D)}{\mu_{L}} \\
\zeta(D) & =\frac{s_{0}(D)\left[\mu_{r}(D)-k_{R} \delta_{r}(D)\right]}{\mu_{L}+k_{L} \delta_{L}}
\end{aligned}
$$

The size effects on $\mu_{r}(D)$ and $\delta_{r}(D)$ can be approximated by Eqs. 11 and 12, where the relevant parameters can be related to $m, \psi$ and $\omega$. The essential step now is to determine $s_{0}(D)$ that would correspond to a prescribed failure risk $P_{c}$.

First consider small-size structures as one limiting case. Based on the finite weakest link model, the strength cdf can be approximated as the Gaussian distribution. Since here we consider that $\sigma_{L}$ follows a Gaussian cdf, it is expected that the Cornell index would give a good estimation of the failure probability, which can be written as

$$
\beta_{c}=\frac{s_{0}(D) \mu_{r}(D)-\mu_{L}}{\sqrt{s_{0}^{2}(D) \delta_{r}^{2}(D)+\delta_{L}^{2}}}
$$

where $\beta_{c}=-\Phi^{-1}\left(P_{c}\right)$. Following [14], we may further write $\sqrt{s_{0}^{2}(D) \delta_{r}^{2}(D)+\delta_{L}^{2}} \approx$ $\epsilon\left[s_{0}(D) \delta_{r}(D)+\delta_{L}\right]$ and $\epsilon$ is around 0.75 for most cases. With such a simplified approximation, we may compute $s_{0}(D)$ as

$$
s_{0}(D)=\frac{\epsilon \beta_{c} \delta_{L}+\mu_{L}}{\mu_{r}(D)}\left[1-\frac{\epsilon \beta_{c} \delta_{r}(D)}{\mu_{r}(D)}\right]^{-1}
$$

At the large-size limit, the probability distribution of structural strength follows the Weibull distribution, which has a power-law tail extending to the point corresponding to a probability of 0.1 (with $5 \%$ error in terms of the probability), i.e. $P_{R}\left(\sigma_{N}\right)=1-\exp \left[-N_{e q}\left(\sigma_{N} / s_{0}\right)^{m}\right] \approx$ $N_{e q}\left(\sigma_{N} / s_{0}\right)^{m}$. Since in practice we are only interested in a low structural failure probability $\left(P_{c} \sim 10^{-6}\right)$, only the left tail matters for the calculation of the overall failure probability:

$$
P_{f}=\int_{0}^{\infty} f_{L}(x) N_{e q}\left(x / s_{0}\right)^{m} \mathrm{~d} x
$$

Therefore, to ensure a failure probability $P_{c}$, we must have

$\mu_{r}(D) s_{0}(D)=\left[P_{c}^{-1} \int_{0}^{\infty} f_{L}(x) x^{m} \mathrm{~d} x\right]^{1 / m} \Gamma(1+1 / m)$

To asymptotically match the aforementioned small and large-size asymptotes, the following approximate equation is proposed for $\mu_{r}(D) s_{0}(D)$ :

$$
\mu_{r}(D) s_{0}(D)=C\left[1+\frac{\gamma \beta_{c} \epsilon \delta_{r}(D)}{\mu_{r}(D)}\right]^{1 / \gamma}
$$

where $C, \gamma$ are constants; $C$ and $\gamma$ can be determined by matching the small-size and large-size asymptotic behavior of $\mu_{r}(D) s_{0}(D)$. With Eqs. 14,15 and 20 , the size-dependent central and nominal safety factors are obtained:

$$
\begin{gathered}
\bar{\zeta}(D)=\mu_{L}^{-1} C\left[1+\frac{\gamma \beta_{c} \epsilon \delta_{r}(D)}{\mu_{r}(D)}\right]^{1 / \gamma} \\
\zeta(D)=C\left[1+\frac{\gamma \beta_{c} \epsilon \delta_{r}(D)}{\mu_{r}(D)}\right]^{1 / \gamma} \frac{1-k_{R} \delta_{r}(D) / \mu_{r}(D)}{\mu_{L}+k_{L} \delta_{L}}
\end{gathered}
$$

\section{ANALYSIS OF FAILURE OF MALPAS- SET DAM}

We now demonstrate the role of size effect in the reliability-based analysis and design of concrete structures by analyzing the failure of Malpsset arch dam. The dam was built across the canyon of the Reyran Valley in France in 1954, and it failed in 1959 at the first full filling 
after five days of heavy rain. It has generally been accepted that the failure was due to the development of vertical flexural cracks engendered by the lateral displacement of the abutment [2, 26].

\subsection{Model Description}

Following the previous study [11], we employ a two dimensional arch model to represent the dam (Fig. 4), in which the dimension of the arch corresponds to the mid-height crosssection of the actual dam, i.e. $R_{a}=92.68$ $\mathrm{m}, D_{a}=6.78 \mathrm{~m}$, and $\alpha=66.5^{\circ}$. The arch is loaded by a displacement at its right support. To investigate the size effect on the failure statistics of the dam, we consider a series of geometrically similar arches with different sizes, where $D=$ arch depth and $R=$ arch radius $=13.67 \mathrm{D}$. The classical engineering beam theory yields the elastic bending stress

$$
\sigma(\theta, \xi)=\sigma_{N} \frac{2 \xi(\cos \theta-\cos \alpha)}{1-\cos \alpha}
$$

where $\xi=x / D$ and $\sigma_{N}=$ structural strength of the arch dam, which is equal to the maximum elastic stress can be reached in the arch. The probability distribution of $\sigma_{N}$ can be calculated using the finite weakest link model (Eq. 1).

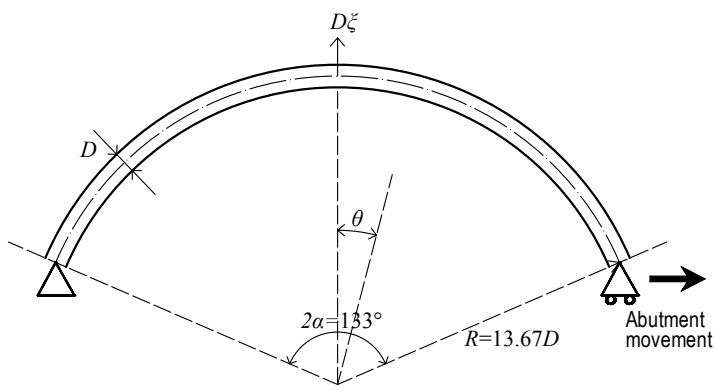

Figure 4: 2D simplified model of dam.

Meanwhile, Castigliano's theorem is used to calculate the nominal applied stress in the arch as a function of the abutment movement $\Delta$ :

$$
\sigma_{L}=\frac{E(1-\cos \alpha) D \Delta}{2 R^{2} \int_{-\alpha}^{\alpha}(\cos \theta-\cos \alpha)^{2} \mathrm{~d} \theta}
$$

Based on the geometry of the dam and elastic property of concrete, we obtain $\sigma_{L}=1.51 \delta$, where $\delta=$ normalized abutment displacement $=\Delta / D$.

The failure state for the arch can simply be written as $\sigma_{N}-\sigma_{L}<0$. Previous studies [11,25] led to the following parameters for the RVE strength distribution of concrete: $m=$ $24, s_{0}=2.12 \mathrm{MPa}, \mu_{G}=2.91 \mathrm{MPa}, \delta_{G}=0.44$ $\mathrm{MPa}, \omega=0.15$, and a RVE size of $280 \mathrm{~mm}$. However, the cdf of $\sigma_{L}$, which is determined by the statistics of abutment movement, is currently not available. In this study we estimate the cdf of $\sigma_{L}$ by assuming: 1) $\sigma_{L}$ follows a Gaussian distribution with a $\mathrm{CoV}$ of $40 \%$, and 2) the failure probability of the dam with a laboratory test scale (i.e. $D=4 l_{0}$ ) is equal to $10^{-6}$, i.e., we assume that the dam was designed on the basis of a laboratory prototype. Based on these assumptions, we obtain the mean value of $\sigma_{L}$ to be $0.76 \mathrm{MPa}$.

\subsection{Discussion based on Cornell index}

According to the aforementioned statistics of $\sigma_{N}$ and $\sigma_{L}$, we can use Eq. 7 to calculate the exact failure probability $P_{f}$ for geometrically similar dams of six sizes $D=$ $4 l_{0}, 8 l_{0}, 16 l_{0}, 24 l_{0}, 48 l_{0}$ and $96 l_{0}$. Note that $D=$ $24 l_{0}$ is the actual size of the Malpsset dam $D_{a}$. As shown in Fig. 5, the structural failure probability increases by three orders of magnitudes as the dam size $D$ increases from $4 l_{0}$ to $96 l_{0}$. We now compare these exact failure probabilities with the predictions by using the sizedependent Cornell index.

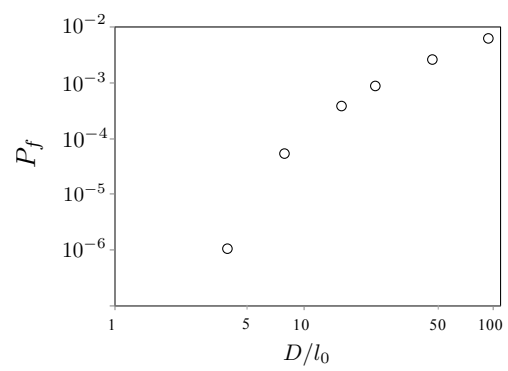

Figure 5: Dependence of failure probability on dam size.

To compute the Cornell reliability index $\beta$, 
we first obtain the size effect on the mean and variance of $\sigma_{N}$ (Eqs. 11 and 12): $N_{a}=0.25$ $\mathrm{MPa}^{0.52} \cdot \mathrm{m}, N_{b}=5312.4 \mathrm{MPa}^{12} \cdot \mathrm{m}, r=0.52$, $C_{a}=0.12 \mathrm{MPa}^{1.48} \cdot \mathrm{m}, C_{b}=1.98 \times 10^{-12}$ $\mathrm{MPa}^{12} \cdot \mathrm{m}$ and $q=0.74$. Fig. 6 compares Eqs. 11 and 12 with the exact mean value and variance of $\sigma_{N}$ calculated from the weakest link model. From the calibrated Eqs. 11 and 12, we can calculate the Cornell index based on Eq. 13 as well as the structural failure probabilities for different dam sizes.
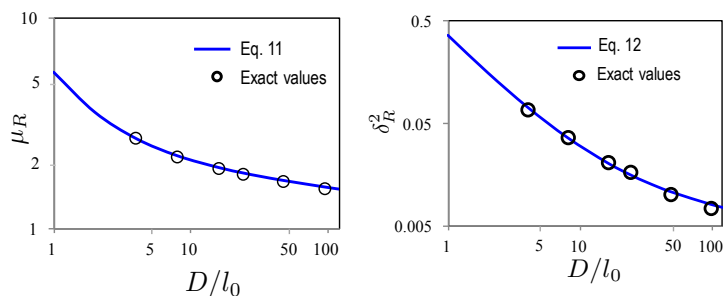

Figure 6: Approximated size effects on mean and variance of nominal strength.

Table 1: Comparison of calculated structural failure probabilities $P_{f}$

\begin{tabular}{|c|c|c|}
\hline Dam Size & Exact $P_{f}$ & $\Phi(-\beta)$ \\
\hline$D=4 l_{0}$ & $1.05 \times 10^{-6}$ & $1.28 \times 10^{-6}$ \\
$D=8 l_{0}$ & $5.37 \times 10^{-5}$ & $2.66 \times 10^{-5}$ \\
$D=16 l_{0}$ & $3.84 \times 10^{-4}$ & $2.04 \times 10^{-4}$ \\
$D=24 l_{0}$ & $8.72 \times 10^{-4}$ & $5.11 \times 10^{-4}$ \\
$D=48 l_{0}$ & $2.57 \times 10^{-3}$ & $1.81 \times 10^{-3}$ \\
$D=96 l_{0}$ & $6.12 \times 10^{-3}$ & $4.89 \times 10^{-3}$ \\
\hline
\end{tabular}

Table 1 compares the exact failure probabilities and those calculated from the Cornell index (i.e. $P_{f}=\Phi(-\beta)$ ). It can be seen that the Cornell index gives a reasonable estimation of the failure probability even though the probability distribution of $\sigma_{N}$ is not Gaussian. To explain this observation, we plot the value of the integrand of Eq. 7 normalized by the failure probability, i.e. $P_{R}(x) f_{L}(x) / P_{f}$, for all dam sizes as shown in Fig. 7. When the structure size is small, the majority of $P_{R}(x)$ follows a Gaussian cdf and the power-law tail makes a negligible contribution to the overall structural failure probability. Therefore, the Cornell index is expected to give a good estimation of the overall failure probability at the small-size limit.

At the large-size limit, Fig. 7 indicates that almost the entire $P_{R}(x)$ contributes to the structural failure probability. Although $P_{R}(x)$ would follow a Weibull distribution, note that, for the same mean and variance, the Gaussian and Weibull distributions are close to each other except for the left and right tails [9]. Therefore, the Cornell index is also able to predict the failure probability reasonably well. Based on the Table 1, it can be seen that, for the intermediate size range, the failure probabilities predicted by the Cornell index are within the same order magnitude of the exact values. Here it should be emphasized that the foregoing analysis is based on the assumption that the applied nominal stress $\sigma_{L}$ follows a Gaussian distribution. For a non-Gaussian distribution of $\sigma_{L}$, the Cornell index may not able to lead to a reasonable estimation of $P_{f}$.

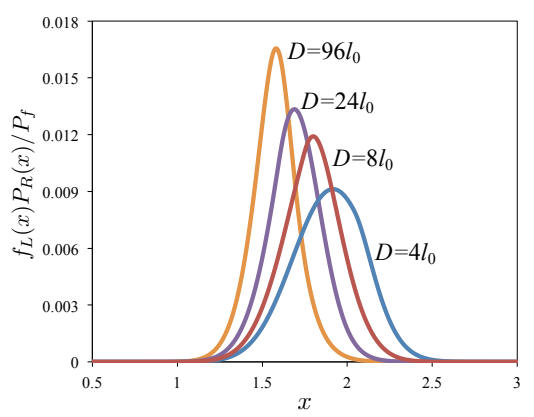

Figure 7: Normalized integrand of Eq. 7.

\subsection{Discussion on Central and Nominal Safety Factors}

The foregoing analysis shows that the structural failure probability of the dam increases significantly with its size. We now calculate the central and nominal safety factors $(\bar{\eta}$ and $\eta)$ for these geometrically similar dams to ensure a failure probability of $P_{c}=10^{-6}$ regardless of the dam size.

In this study, we set the following parameters for computing $\bar{\eta}$ and $\eta$ : the Weibull modulus $m=24$, the CoV of RVE strength $\omega=0.15$, 
and the ratio between the mean RVE strength and the Weibull scaling parameter $\psi=\mu / s_{0}=$ 1.37. The Weibull scaling parameter $s_{0}$ of RVE strength distribution is determined so that the overall structural failure probability be equal to $10^{-6}$. We do so by using Eq. 7. Then we can compute the mean structural strength and the corresponding values of $\bar{\zeta}$ and $\zeta$ for different dam sizes. The exact values of $\bar{\zeta}$ and $\zeta$ are shown by the circles in Figs. $8 \mathrm{a}$ and $\mathrm{b}$.
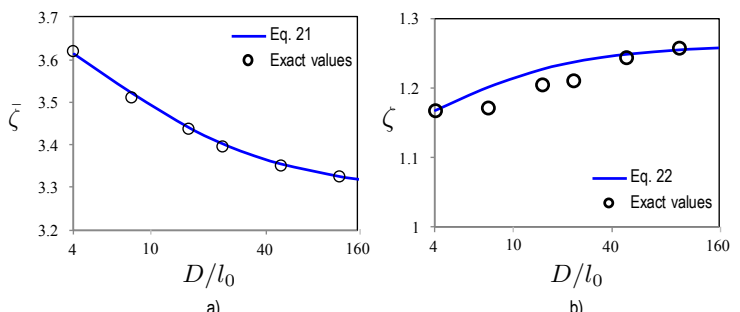

Figure 8: Calculated size effects on central and nominal safety factors.

Eqs. 21 and 22 are now used to compute the size dependence of central and nominal safety factors. To use Eq. 21, we first determine functions $\mu_{r}(D)$ and $\delta_{r}^{2}(D)$, which describe the size effect on the mean and variance of the structural strength of the dam made of an artificial RVE with the following statistical parameters: $m=24, s_{0}=1, \mu_{G}=1.37$ and $\omega=0.15$. By matching the small- and large-size asymptotes of Eq. 21 with Eqs. 17 and 19, we obtain $C=2.835 \mathrm{MPa}$ and $\gamma=2.4$. Fig. 8a shows that the central safety factors predicted by Eq. 21 agree well with the exact values for all dam sizes. It is interesting to observe that the central safety factor decreases as the structural size increases. This is because, according to Eq. 21, the size dependence on the central safety factor is governed by the size effect on the $\mathrm{CoV}$ of $\sigma_{N}$, which has a decreasing trend in the present analysis. At the large size limit, the structural strength follows a Weibull distribution and, therefore, the CoV becomes invariant with the structure size. Therefore, the size dependence of the central safety factor will vanish as the structure size is sufficiently large.

For the nominal safety factor, we choose
$k_{R}=k_{L}=3$. The prediction of Eq. $22 \mathrm{com}-$ pares well with the exact values of $\zeta$ in Fig. 8b. In contrast to the size effect on the central safety factor, $\zeta$ increases with the structure size. The reason is that the decreasing in $\mathrm{CoV}$ of $\sigma_{N}$ has two effects on the nominal safety factor: 1) it leads to a lower central safety factor, and 2) the ratio between the nominal and mean values of the structural strength becomes larger. As suggested by Eq. 21, these two effects counter each other. For the current $k_{R}$ value, effect 2 is more dominant, which leads to an increasing $\zeta$ with the structure size. If we choose a smaller $k_{R}$ value, effect 1 would prevail and $\zeta$ would then decrease with the structure size. Overall speaking, due to the competition between these two effects, the size effect on the nominal safety factor is less pronounced compared to that on the central safety factor.

\section{CONCLUSIONS}

1. The finite weakest link model correctly captures the size dependence of strength distribution of quasibrittle structures, which transitions from a grafted Gaussian-Weibull cdf at the small size limit to a Weibull cdf at the large size limit. The model agrees well with the measured strength histograms of various quasibrittle materials, such as engineering, dental ceramics, fiber composites and cold asphalt mixtures.

2. By assuming that the applied load follows a the Gaussian distribution, it is shown that the Cornell index may give a reasonable estimation of the failure probability of quasibrittle structures. The scale effect on the Cornell index can be obtained directly from the approximate size effect equations on the mean and variance of the structural strength.

3. Approximate size effect equations are derived for the central and nominal safety factors by asymptotic matching. It is shown that the size dependence of the safety factors is governed by the size effect on the coefficient of variation of the structural strength, and the size effect on the central safety factor is more pronounced than that on the nominal safety factor. Such size dependence plays a critical role in 
the interpretation of small-scale laboratory testing for the reliability-based design of full-scale structures.

\section{REFERENCES}

[1] Ang, A.H.-S., and Tang, W.H. (1984). Probability Concepts in Engineering Planning and Design. Vol II. Decision, Risk and Reliability. J. Wiley, New York.

[2] Bartle, A., Ed., (1985) "Four major dam failures re-examined." Int. Water Power Dam Constr., 37(11), 33-36, 41-46.

[3] Bažant, Z.P. (1984). "Size effect in blunt fracture: Concrete, rock, metal." J. of Engrg. Mechanics, ASCE, 110 (4), 518-535.

[4] Bažant, Z. P. (2004) "Scaling theory of quaisbrittle structural failure." Proc. Nat'l. Acad. Sci., USA 101 (37), 13397-13399.

[5] Bažant, Z. P. (2005) Scaling of Structural Strength, 2nd Ed., Elsevier, London.

[6] Bažant, Z. P., Le, J.-L., and Bazant, M. Z. (2009). "Scaling of strength and lifetime distributions of quasibrittle structures based on atomistic fracture mechanics." Proc. Nat'l Acad. Sci. USA 11484-11489

[7] Bažant, Z. P., and Novák, D. (2000). "Probabilistic nonlocal theory for quasibrittle fracture initiation and size effect. I. Theory." J. of Engrg. Mech. ASCE 126 (2), 166-174.

[8] Bažant, Z. P., and Pang, S.-D. (2006) "Mechanics based statistics of failure risk of quasibrittle structures and size effect on safety factors." Proc. Nat'l Acad. Sci., USA 103 (25), pp. 9434-9439.

[9] Bažant, Z. P., and Pang, S.-D. (2007) "Activation energy based extreme value statistics and size effect in brittle and quasibrittle fracture." J. Mech. Phys. Solids 55, pp. 91-134.
[10] Bažant, Z. P. and Planas, J. (1998) Fracture and Size Effect in Concrete and Other Quasibrittle Materials, CRC Press.

[11] Bažant, Z. P., Vořechovský, M., and Novak, D. (2007) "Asymptotic prediction of energetic-statistical size effect from deterministic finite element solutions." J. Engrg. Mech, ASCE, 128, 153-162.

[12] Bažant, Z. P. and Xi, Y. (1991) "Statistical size effect in quasi-brittle structures: II. Nonlocal theory." J. Engrg. Mech., ASCE 117(7), 2623-2640.

[13] Cornell, C. A. (1969). "A probabilitybased structural code." J. Amer. Concrete Inst., 66(12), 974?985.

[14] Haldar, A., and Mahadevan, S. (2000) Probability, Reliability, and Statistical Methods in Engineering Design. Wiley, New York.

[15] Bažant, Z.P., and Yu, Qiang (2009). "Does strength testsatisfying code requirement for nominal strength justify ignoringsize effect in shear?" ACI Materials Journal 106 (1, Jan.-Feb.), 14-19.

[16] Haldar, A. and Mahadevan, S. (2000b) Reliability Assessment Using Stochastic Finite Element Analysis. Wiley, New York.

[17] Duckett, K. (2005). "Risk analysis and the acceptable probability of failure." The Structural Engineering 83 (15), pp 25-26.

[18] Grassl, P., and Bažant, Z. P. (2009). "Random lattice-particle simulation of statistical size effect in quasi-brittle structures failing at crack initiation." J. of Engrg. Mech. ASCE 135 (2), Feb., 85-92.

[19] Gross, B. (1996) "Least squares best fit method for the three parameter weibull distribution: analysis of tensile and bend specimens with volume or surface flaw failure." NASA TM-4721 pp 1-21. 
[20] Gumbel, E.J. (1958). Statistics of Extremes. Columbia University Press, New York.

[21] Kramers H. A. (1941) "Brownian motion in a field of force and the diffusion model of chemical reaction." Physica 7, 284304.

[22] Le, J.-L (2015) "Size effect on reliability indices and safety factors of quasibrittle structures." Structural Safety, 52. 20-28.

[23] Le, J.-L., Bažant, Z. P. and Bazant, M. Z. (2011). "Unified nano-mechanics based probabilistic theory of quasibrittle and brittle structures: I. Strength, crack growth, lifetime and scaling". J. Mech. Phys. of Solids, 59, 1291-1321.

[24] Le, J.-L., Cannone Falchetto, A. and Marasteanu, M. O. (2013) "Determination of strength distribution of quasibrittle structures from size effect analysis", $M e$ chanics of Materials, 66, 79-87.

[25] Le, J.-L., Eliášs, J. and Bažant, Z. P. (2012) "Computation of probability distribution of strength of quasibrittle structures failing at macro-crack initiation", J. Engrg. Mech., ASCE, 138, No. 7, 888-899.

[26] Levy, M., and Salvadori, M. (1992) Why Buildings Fall Down?, W. W. Norton, New York.
[27] Melchers, R. E. (1987) Structural Reliability, Analysis \& Prediction. Wiley, New York.

[28] NKB (1978) (Nordic Committee for Building Structures). Recommendation for loading and safety regulations for structural design. NKB Report, No. 36.

[29] Tsai, S. W. and Wu, E. M. (1971) “A general theory of strength for anisotropic materials." Journal of Composite Materials, 5 , pp. $58-80$

[30] Santos, C. d., Strecker, K., Piorino Neto, F., Oliveério Moreira de Macedo Silva, Sandro Aparecido Baldacum and Cosme Roberto Moreira da Silva. (2003) "Evaluation of the reliability of $\mathrm{Si}_{3} \mathrm{~N}_{4}-\mathrm{Al}_{2} \mathrm{O}_{3}$ $\mathrm{CTR}_{2} \mathrm{O}_{3}$ ceramics through Weibull analysis." Materials Research Vol.6, No.4, pp. 463-467.

[31] Tinschert, J., Zwez, D., Marx, R. and Ausavice, K. J. (2000). "Structural reliability of alumina-, feldspar-, leucite, mica- and zirconia-based ceramics." $J$. Dent. Vol 28, pp. 529-535.

[32] Weibull, W. (1939) "The phenomenon of rupture in solids." Proc., Royal Swedish Inst. Eng. Res. 153, Stockholm, 1939, 155.

[33] Weibull, W. (1951) “ A statistical distribution function of wide applicability." $J$. Appl. Mech. ASME, 153(18), 293-297. 